\title{
Alignment of liquid crystals on a photosensitive substrate studied by surface optical second-harmonic generation
}

\author{
I. Drevenšek Olenik, ${ }^{1}$ M. W. Kim, ${ }^{2,3}$ A. Rastegar, ${ }^{2,4}$ and Th. Rasing ${ }^{2}$ \\ ${ }^{1}$ Faculty of Mathematics and Physics, University of Ljubljana, Jadranska 19, SI 1000, Ljubljana, Slovenia \\ and J. Stefan' Institute, Jamova 39, SI 1001 Ljubljana, Slovenia \\ ${ }^{2}$ Research Institute for Materials, University of Nijmegen, Toernooiveld, 1, 6525 ED, Nijmegen, The Netherlands \\ ${ }^{3}$ Department of Advanced Materials Engineering, Korea Advanced Institute of Science and Technology, Seoul, Korea \\ ${ }^{4}$ Nijmegen SON Research Institute for Molecular Structures, Design and Synthesis, University of Nijmegen, Toernooiveld 1, \\ 6525 ED, Nijmegen, The Netherlands
}

(Received 19 July 1999; revised manuscript received 6 October 1999)

\begin{abstract}
The adsorption kinetics and orientational ordering of $4^{\prime}$ - $n$-octyl-4-cyanobiphenyl (8CB) liquid crystal films evaporated onto photosensitive poly(vinyl-cinnamate) $(\mathrm{PVCN})$ substrates were investigated by surface optical second-harmonic generation. The adsorption rate of the first monolayer decreases with increasing degree of photochemical modification of the polymer. The in-plane orientational anisotropy of the 8CB films grown on unidirectionally photopolymerized PVCN substrates is considerably lower than the intrinsic surface orientational anisotropy of these substrates, which can explain the generally found weak surface anchoring of liquid crystals on PVCN alignment layers.
\end{abstract}

PACS number(s): 42.70.Df, 42.70.Gi, 78.66.Qn, 68.45.Da

During the last decade several techniques have been developed for liquid crystals (LC) alignment by photosensitive polymeric layers, opening up new possibilities for patterning and switching of liquid crystal displays (LCD's) [1-5]. A planar alignment is achieved using linearly polarized light that causes directionally selective photochemical transformations. The resulting unidirectional structure induces the LC alignment presumably via anisotropic van der Waals forces [2], similar to the alignment of liquid crystals on conventionally rubbed polymeric layers [6,7].

Despite this similarity, generally there are large differences found between the surface anchoring strengths of these two types of substrates. Rubbed polymers, such as polyimides, usually give strong azimuthal surface anchoring energy coefficients $W \varphi>10^{-4} \mathrm{Jm}^{-2}$ [8]. In contrast, unidirectionally photo-modified polymers such as photoresists and various kinds of azo-polymers give weak surface anchoring with $W \varphi<10^{-5} \mathrm{Jm}^{-2}$ [9-11]. Yet the induced optical birefringence $\Delta n$ of both types of substrates appears to be of the same order of magnitude $[9,10,12,13]$, which is presumably connected with the fact that for a rubbed polymer, the anisotropy originates from a thin surface layer, whereas the photopolymerization is a bulk effect.

However, recent surface second-harmonic generation (SSHG) and optical birefringence experiments on poly(vinyl cinnamate) (PVCN) have shown that its surface and its bulk photoreaction kinetics and photoinduced anisotropy are very similar $[14,15]$. The maximum detected value of the surface in-plane orientational order parameter of the trans-cinnamoyl side groups was $Q_{s}=0.15$, which is close to the usual values of $Q_{s} \sim 0.3-0.5$ for rubbed polymeric surfaces [16-18]. This suggests that the weak surface anchoring of liquid crystals on PVCN is not due to its low surface anisotropy, but arises from a different alignment mechanism.

To clarify these aspects we performed SSHG measurements to probe the relation between the intrinsic surface an- isotropy of the PVCN substrates and the surface anisotropy of the adjacent $44^{\prime}$-n-octyl-4-cyanobiphenyl (8CB) LC monolayer deposited onto them. SSHG derives its surface sensitivity from the symmetry breaking at interfaces and is therefore extremely suitable to probe these surface properties [20]. We found that the growth rate of the first $8 \mathrm{CB}$ monolayer strongly decreased with increasing degree of photopolymerization. The azimuthal dependences of the SSHG showed that the in-plane orientational order parameter of the adsorbed 8CB films $Q_{s}^{m} \leqslant 0.02$ was much lower than the initial in-plane orientational order parameter of the substrates. The observed reduction of the surface adhesion and orientational ordering can be explained by a reduced density of the trans-cinnamoyl side groups, which seem to play a crucial role in the interaction of the PVCN with LC's.

The photopolymeric substrates were prepared as described in our previous paper [15]. For the SSHG measurements, we used a Ti:sapphire laser ( $76 \mathrm{MHz}, 100 \mathrm{fs}$ pulses) at the fundamental wavelength of $800 \mathrm{~nm}$, with an average power of $500 \mathrm{~mW}$ at an incident angle of $45^{\circ}$, focused to a spot size of about $100 \mu \mathrm{m}$. After proper spatial and spectral filtering the outcoming specularly reflected SHG light was detected by a photomultiplier in connection with a photon counter. The PVCN substrate was mounted on a rotation stage in air. The $8 \mathrm{CB}$ (Merck Ltd.) was evaporated onto the substrate from a hot plate $\left(T \sim 100^{\circ} \mathrm{C}\right)$ positioned $5 \mathrm{~cm}$ below the substrate, while the SSHG signal was used to monitor the adsorption. Before and after the evaporation the SSHG signal from the samples was practically constant (decrease below $10 \%$ per hour), confirming that there was no essential surface bleaching of the samples due to the laser irradiation and no significant desorption of the $8 \mathrm{CB}$.

Figure 1 shows the dependence of the SSHG power $P(2 \omega)$ on evaporation time of $8 \mathrm{CB}$ on an unexposed PVCN substrate and on one that was exposed to unpolarized uv light for $20 \mathrm{~min}$. The unexposed substrate shows a strong 


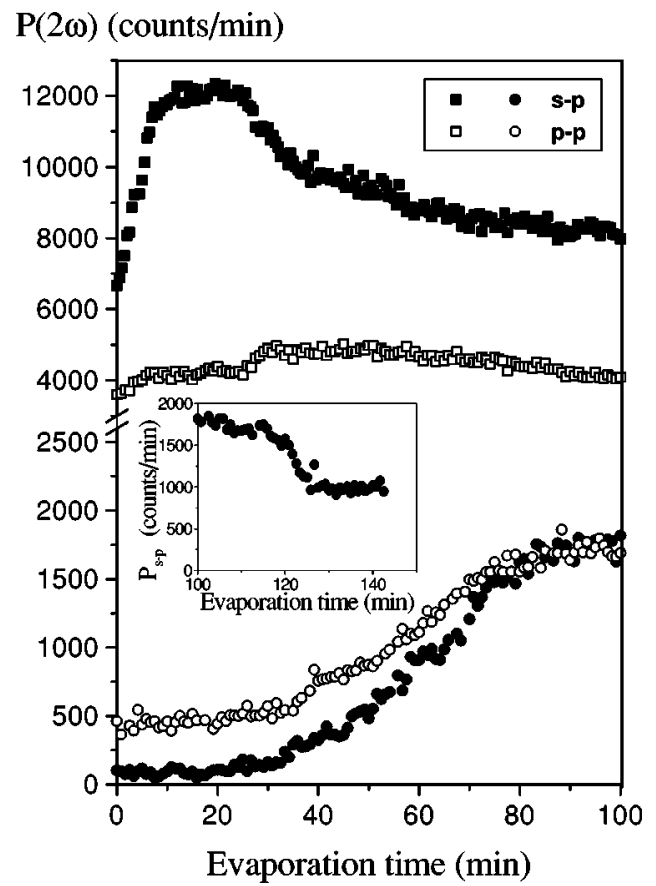

FIG. 1. Second-harmonic signal during the evaporation of $8 \mathrm{CB}$ on the unexposed PVCN substrate (squares) and on the PVCN substrate exposed to unpolarized uv light for $20 \mathrm{~min}$ (circles).

increase in the $s-p$ polarization combination and a much weaker but similar behavior for $p-p$ (here $m-n$ indicate the polarizations of the fundamental and second harmonic beams, respectively). The initial almost linear increase is followed by a plateau and later on by a steplike change. Further deposition leads to a gradually decreasing dependence for long evaporation times. The results for the uv exposed substrate are significantly different. We have found before that such exposures leads to a practically complete phototransformation of the cinnamoyl side chains [15]. The background SSHG signal from the uv exposed substrate is about an order of magnitude lower than from the unexposed substrate and appears predominantly in the $p-p$ polarization combination, which is a reversal of the situation for the unexposed sample. During the evaporation the $s-p$ and $p-p$ signals both strongly increase and then reach a plateau of similar magnitude. The evaporation time corresponding to the plateau is about ten times longer than the corresponding one for the unexposed substrate. The inset of Fig. 1 shows again a steplike decrease of $P_{s-p}$ for $t>100 \mathrm{~min}$. Similar results were found for other polarization combinations. The values of $P_{p-s}$ and $P_{s-s}$ were in general very low, in accordance with the $C_{\infty v}$ symmetry of the samples.

The second order surface nonlinear optical susceptibility tensor $\chi_{s}^{(2)}$ of the $8 \mathrm{CB}$ coated PVCN substrate can in general be expressed as

$$
\chi_{s}^{(2)}=\chi_{0}+\chi^{m}+\chi_{i n t},
$$

where $\chi_{0}$ denotes the substrate response, $\chi^{m}$ the susceptibility of the adsorbed molecules, and $\chi_{\text {int }}$ a possible interaction between the substrate and the adsorbed layer. The latter will be neglected, as it is expected to be weak (van der Waals) [2]. The reflected SSHG power $P(2 \omega)$ is given by [20]

$$
\begin{aligned}
P(2 \omega) & =A\left|[L \hat{e}(2 \omega)] \chi_{s}^{(2)}:[L \hat{e}(\omega)][L \hat{e}(\omega)]\right|^{2} P^{2}(\omega) \\
& =A\left|\chi_{e f f}\right|^{2} P^{2}(\omega)
\end{aligned}
$$

where $P(\omega)$ is the power of incident fundamental beam, $\hat{e}(\omega)$ and $\hat{e}(2 \omega)$ are polarization vectors of the incident and the second harmonic beam respectively, $L$ is the Fresnel transformation matrix [21], and $A=\omega^{2} \sec ^{2} \Omega / 2 \varepsilon_{0} c^{3} S$ with $\Omega=45^{\circ}$ the angle of incidence and $S$ the spot size of the beam.

Because the incident and SHG wavelengths are away from the absorption bands of the PVCN and 8CB, $\chi_{0}$ and $\chi^{m}$ may be taken to be real. With these assumptions $\chi_{\text {eff }}$ of an adsorbed $8 \mathrm{CB}$ film can be calculated as

$$
\left|\chi_{e f f}^{m}\right| \propto|\sqrt{P(2 \omega)} \mp \sqrt{P(2 \omega, t=0)}|,
$$

where $P(2 \omega)$ is the SSHG from the substrate covered with $8 \mathrm{CB}$ and $P(2 \omega, t=0)$ is the signal from the substrate. The sign $(+/-)$ of the relative phase of these two contributions follows from the variation of $P(2 \omega, t)$ during evaporation. In Fig. $1 P(2 \omega, t)$ originally increases monotonically with time. This suggests that the contributions from the PVCN substrate and from the $8 \mathrm{CB}$ layer add constructively (in-phase), so that the minus sign is relevant in Eq. (3).

The first plateau in $P(2 \omega, t)$ is generally assumed to coincide with the formation of the first complete monolayer of the LC molecules [19]. The analysis of the corresponding SSHG data thus provides information on the nonlinear susceptibility $\chi^{m}$ of this monolayer. From the observed variations of the $P_{s-p}$ and $P_{p-p}$ on several samples, the following ratios for the effective susceptibilities were deduced: $\left(\chi_{e f f, s-p}^{m} / \chi_{e f f, p-p}^{m}\right)=5.5 \pm 2.4$ for the 8CB on the unexposed substrate, and $\left(\chi_{e f f, s-p}^{m} / \chi_{e f f, p-p}^{m}\right)=1.6 \pm 0.3$ for the $8 \mathrm{CB}$ on the substrate that was exposed to unpolarized uv light for 20 $\min$.

Assuming the component $\beta_{333}$ of the molecular hyperpolarizability along the long molecular axis $\hat{e}_{3}$ of $8 \mathrm{CB}$ to be strongly dominant and assuming a $\delta$ function distribution of the molecular tilt angles $f_{s}(\theta)=\delta\left(\theta-\theta_{0}\right)[19,21]$, these observed ratios result in $\theta_{0}=65^{\circ} \pm 2^{\circ}$ for $8 \mathrm{CB}$ on the unexposed and $\theta_{0}=76^{\circ} \pm 5^{\circ}$ for $8 \mathrm{CB}$ on the uv exposed PVCN substrate. The photomodification thus increases the surface tilt angle of the molecules in the adjacent $8 \mathrm{CB}$ monolayer. The obtained value of $\theta_{0} \sim 76^{\circ}$ is very similar to the tilt angles of ciano-biphenyl molecules usually found on substrates that induce a planar alignment $[7,21]$.

The magnitudes of $P_{s-p}$ corresponding to the plateau for the unexposed and for the unpolarized uv exposed substrate were used to calculate the ratio between the corresponding values of the surface density $N_{s}$ that was found to be 1:4. Note that $N_{s}$ actually only gives the density of the polar oriented $8 \mathrm{CB}$ molecules, while the molecules attached to the surface in head to tail "pairs" do not contribute to the SSHG signal [19]. The magnitude of $N_{s}$ is thus more a measure for the surface polarity than for the surface coverage of the substrate. This suggests that the photochemical reactions increase the surface polarity of the PVCN. The latter may be explained with the conformational modifications of the cinnamoyl side chains, which increase the net surface dipole 


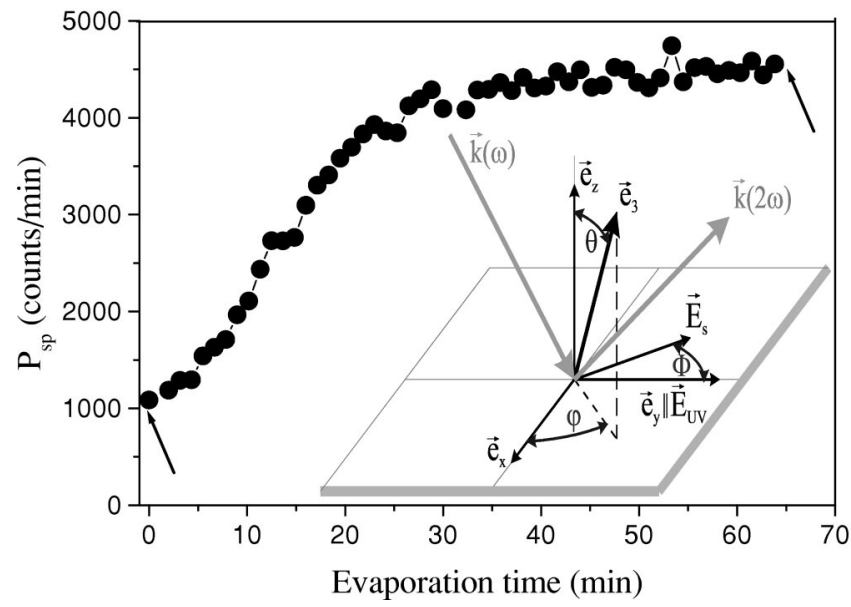

FIG. 2. Dependence of $P_{s-p}$ on the evaporation time of 8CB on the PVCN substrate that was exposed to linearly polarized uv light for $60 \mathrm{~min}$. Arrows indicate the moments at which azimuthal dependences (Fig. 3) were also measured. The inset shows the orientation of various coordinate axes of the sample, the direction of the uv polarization, and the direction of the $s$ polarized optical field.

moment of the polar carbonyl groups. Figure 1 also shows a prominent difference in the adsorption kinetics for the two different substrates, but this will be discussed in a separate paper.

Figure 2 shows the time dependence of the $P_{s-p}$ during evaporation of the $8 \mathrm{CB}$ onto a 60 min exposed linearly photopolymerized (LPP) substrate. The polarization of the uv light was parallel to the $s$ polarization of the laser beam (see the inset). Because the LPP process is not yet completed within $60 \mathrm{~min}$ [15], the initial signal from the substrate $P_{s-p}(t=0)$ is larger than the $P_{s-p}(t=0)$ for the uv exposed substrate in Fig. 1. The first plateau in the $P_{s-p}(t)$ is observed at about $30 \mathrm{~min}$, which is as well somewhere in-between the two cases shown in Fig. 1.

Figure 3 shows the azimuthal dependences of the $P_{s-p}$ and $P_{p-p}$ of this sample, where $\Phi=0^{\circ}$ corresponds to the incident $s$ polarization being parallel to the direction of the uv polarization (see the inset of Fig. 2). Two cases are shown: a bare LPP substrate prepared by $60 \mathrm{~min}$ of LP uv exposure and the same substrate coated with an $8 \mathrm{CB}$ monolayer. The LP uv irradiation induces an apparent anisotropy of $P_{s-p}(\Phi)$ and $P_{p-p}(\Phi)$. After the 8CB adsorption the anisotropy is still notable, but its magnitude is considerably reduced. This suggests that the additional SSHG contribution from the $8 \mathrm{CB}$ film is more or less isotropic.

In accordance with the expected $C_{2 v}$ surface symmetry of the $8 \mathrm{CB}$ monolayer on a LPP substrate, its nonlinear optical susceptibility $\chi^{m}$ can be described by three independent nonzero components [22],

$$
\begin{gathered}
\chi_{z z z}^{m}=N_{s}\left\langle\cos ^{3} \theta\right\rangle \beta_{333}, \\
\chi_{z x x}^{m}=\chi_{x z x}^{m}=N_{s}\left\langle\sin ^{2} \theta \cos \theta \cos ^{2} \varphi\right\rangle \beta_{333}, \\
\chi_{z y y}^{m}=\chi_{y z y}^{m}=N_{s}\left\langle\sin ^{2} \theta \cos \theta \sin ^{2} \varphi\right\rangle \beta_{333},
\end{gathered}
$$

where $\theta$ and $\varphi$ describe the relative orientation of the molecular long axis $\hat{e}_{3}$ with respect to the surface normal $\hat{e}_{z}$ (see
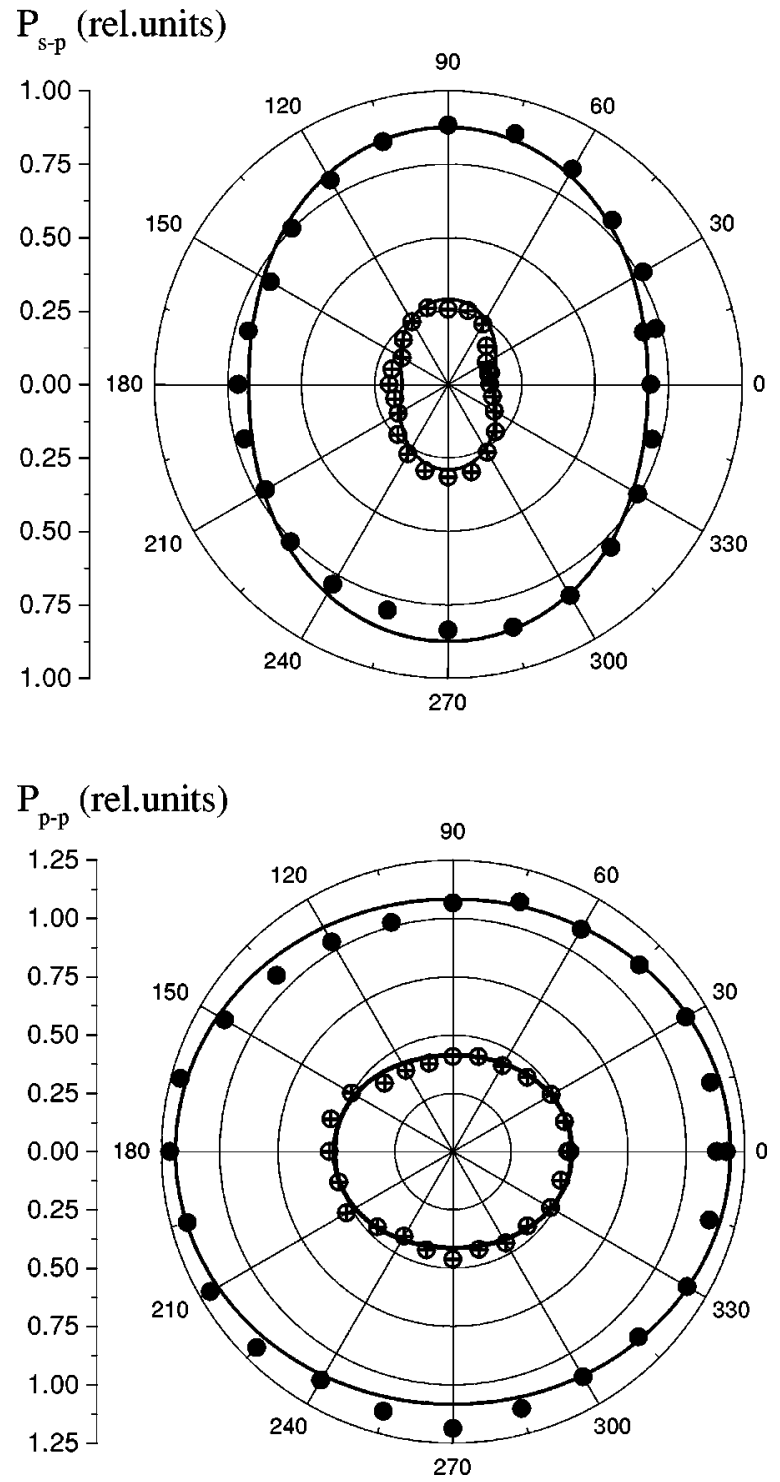

FIG. 3. Dependencies of $P_{s-p}$ and (top) $P_{p-p}$ (bottom) on the sample rotation angle $\Phi: \mu$ bare PVCN substrate after 60 min of LP uv irradiation (crossed circles), and $\mu$ the same substrate with the deposited 8CB monolayer (closed circles). $\Phi=0$ corresponds to the $s$ polarization parallel to the uv polarization (see the inset of Fig. 2). The magnitudes are given relative to the corresponding signal for the unexposed substrate. Solid lines are fits to Eq. (5).

the inset of Fig. 2) and \langle\rangle indicate averaging over their angular distribution. The effective susceptibilities for the $p$ polarized reflected SHG beam are then given as [21]

$$
\chi_{e f f, s-p}^{m}=\left(\chi_{z x x}^{m} \sin ^{2} \Phi+\chi_{z y y}^{m} \cos ^{2} \Phi\right) L_{z z}(2 \omega) L_{y y}^{2}(\omega) \sin \Omega,
$$

$$
\begin{aligned}
\chi_{e f f, p-p}^{m}= & \chi_{z z z}^{m} L_{z z}(2 \omega) L_{z z}^{2}(\omega) \sin ^{3} \Omega+\left(\chi_{z x x}^{m} \cos ^{2} \Phi\right. \\
& \left.+\chi_{z y y}^{m} \sin ^{2} \Phi\right) \cos ^{2} \Omega \sin \Omega \times\left[L_{z z}(2 \omega) L_{x x}^{2}(\omega)\right. \\
& \left.-2 L_{x x}(2 \omega) L_{z z}(\omega) L_{x x}(\omega)\right] .
\end{aligned}
$$

By assuming independent polar and azimuthal parts of the molecular orientational angular distribution function 
$f_{s}(\theta, \varphi)=\delta\left(\theta-\theta_{0}\right) g(\varphi)$, the in-plane surface orientational order parameter $Q_{s}^{m}=\langle\cos 2 \varphi\rangle$ of the 8CB monolayer can be calculated from the $\chi^{m}$ as [7]

$$
Q_{s}^{m}=\left(\chi_{z x x}^{m}-\chi_{z y y}^{m}\right) /\left(\chi_{z x x}^{m}+\chi_{z y y}^{m}\right) .
$$

From our azimuthal SSHG measurements shown in Fig. 3 we deduced the surface in plane order parameter $Q_{s}$ of the LPP PVCN substrate $[14,15]$, and also the surface in plane order parameter $Q_{s}^{m}$ of the 8CB monolayer on top of it. The resulting values were $Q_{s}=0.15$ and $Q_{s}^{m} \sim 0.02$. The latter value is, however, questionable, because it is in the limit of the experimental accuracy of our setup. Therefore we can conclude that the value of the $Q_{s}^{m}$ of the first $8 \mathrm{CB}$ monolayer is probably below 0.02 .

Note that, witin the electric dipole aproximation, SSHG only originates from that part of the interface where the inversion symmetry is broken [21]. For the LC side, this means approximately one monolayer where as for the polymer surface the corresponding "probing depth" is of similar size. The fact that we find $Q_{s}$ of the photopolymerized substrate to be similar to that of a rubbed surface indicates that the degree of alignment is similar in both cases. However, as we have shown before [15], the concentration of the transcinnamoyl side groups is strongly reduced due to their phototransformation, resulting in a low concentration of aligned trans-side groups in a matrix of less effective photoproducts,

[1] W. M. Gibbons, P. J. Shannon, S. T. Sun, and B. J. Swetlin, Nature (London) 351, 49 (1991).

[2] M. Schadt, K. Schmitt, V. Kozinkov, and V. Chigrinov, Jpn. J. Appl. Phys. 31, 2155 (1992).

[3] T. Ya. Marusii and Yu. A. Reznikov, Mol. Mater. 3, 161 (1993).

[4] P. J. Shannon, W. M. Gibbons, and S. T. Sun, Nature (London) 368, 532 (1994).

[5] M. Schadt, H. Seiberle, and A. Schuster, Nature (London) 381, 212 (1996).

[6] J. A. Castelano, Mol. Mater. 94, 33 (1983).

[7] M. Barmentlo, N. A. J. M. van Aerle, R. W. J. Hollering, and J. P. M. Damen, J. Appl. Phys. 71, 4799 (1992).

[8] E. S. Lee, P. Vetter, T. Miyashita, and T. Uchida, Jpn. J. Appl. Phys. 32, L1339 (1993).

[9] G. P. Bryan-Brown and I. C. Sage, Liq. Cryst. 20, 825 (1996).

[10] X. T. Li, D. H. Pei, S. Kobayashi, and Y. Iimura, Jpn. J. Appl. Phys. 36, L432 (1997).

[11] V. P. Vorflusev, H. -S. Kitzerow, and V. G. Chigrinov, Appl. Phys. A: Mater. Sci. Process. 64, 615 (1997).

[12] N. A. J. van Aerle, M. Barmentlo, and R. W. J. Hollering, J. leading to a low value of $W_{\varphi}$. This weak interaction between the cinnamoyl side chains and $8 \mathrm{CB}$ molecules then results in the very low value of $Q_{s}^{m} \leqslant 0.02$.

In conclusion, our results have shown that the photopolymerization of the PVCN strongly affects its surface adhesion characteristics and its azimuthal interaction strength, because it reduces the density of the anisotropic trans-cinnamoyl side groups. Because of this, photoalignment of any polymer will always give a weaker surface anchoring than a conventional rubbing of this polymer. To minimize this disadvantage one should use photopolymers in which the photoreaction products provide a coordinated aligning action on the liquid crystalline molecules.

We have also shown that using a Ti:saphire laser it is possible to obtain a reasonable off resonant SSHG signal from an adsorbed monolayer of LC molecules. This condition is contrary to practically all the SSHG experiments performed on LC's up till now. The off resonance condition leads to a relatively large SSHG signal from the substrate, but simplifies the phase relations between the various contributions, allowing the study of the adsorption and orientational ordering of the LC layers on various substrates in a very convenient way.

Part of this work was supported by the European Network Contract No. ERB FMRXCT 980209 (SILC).

Appl. Phys. 74, 3111 (1993).

[13] N. A. J. M. van Aerle and A. J. W. Tol, Macromolecules 27, 6520 (1994).

[14] I. Drevenšek Olenik, M. W. Kim, A. Rastegar, and Th. Rasing, Appl. Phys. B: Lasers Opt. 68, 599 (1999).

[15] I. Drevenšek Olenik, M. W. Kim, A. Rastegar, and Th. Rasing, Phys. Rev. E 60, 3120 (1999).

[16] X. Wei, X. Zhuang, S. C. Hong, T. Goto, and Y. R. Shen, Phys. Rev. Lett. 82, 4256 (1999).

[17] R. Meister and B. Jérôme, Macromolecules 32, 480 (1999).

[18] T. Sakai, J. G. Yoo, Y. Kinoshita, K. Ishikawa, H. Takezoe, A. Fukuda, T. Nihira, and H. Endo, Appl. Phys. Lett. 71, 2274 (1997).

[19] C. S. Mullin, P. Guyot-Sionnest, and Y. R. Shen, Phys. Rev. A 39, 3745 (1989).

[20] T. F. Heinz, in Nonlinear Surface Electromagnetic Phenomena, edited by H. E. Ponath and G. I. Stegeman (North Holland, Amsterdam, 1991), p. 353.

[21] M. B. Feller, W. Chen, and Y. R. Shen, Phys. Rev. A 43, 6778 (1991).

[22] J. R. Dennis and V. Vogel, J. Appl. Phys. 83, 5195 (1998). 